Pacific Journal of Mathematic 


\section{PROJECTIVE COLORINGS}

\section{A. W. Hales and E. G. Straus}

All colorings of the points of (Desarguesian) projective planes in three colors so that no straight line contains points of all three colors are characterized in terms of the valuations of the field of coordinates. Generalizations to higher dimensions and applications to the Fundamental Theorem of Projective Geometry and the division of polygons into disjoint triangles of equal areas are given. We restrict our discussion to the Pappian (commutative coordinate field) case. For the general division ring case see [3].

1. Introduction. M. Bognár (Budapest University) recently posed the following question: can the points of the real projective plane be colored in three colors (nontrivially) so that no line contains all three colors? Here nontrivial means that no color is confined to one line. For the affine plane this question had been answered in the affirmative in [6]. In this paper we give a complete characterization of these colorings and extend our results in various directions (other fields, higher dimensions, and curves of higher degree). The results involve a nontrivial blending of combinatorics and valuation theory. In $\S 5$, we give a generalization of the fundamental theorem of projective geometry suggested by our results. In $\S 6$ we extend results of [6] to the division of polygons into disjoint triangles of equal areas.

After this paper was written we learned of the work of D. S. Carter and A. Vogt [3]. We wish to acknowledge their priority, especially in the discovery of Theorems 1 and 5 of this paper. In view of our somewhat different approach we give the comparatively short proofs of these two theorems.

2. Colorings of projective planes. Let $\boldsymbol{P}_{2}(F)$ denote the projective plane over the field $F$. (For convenience we assume $F$ to be commutative, although it does not appear necessary for our results. It is not clear, however, whether our results extend to the non-Desarguesian case.) We wish to color the points of $\boldsymbol{P}_{2}(\boldsymbol{F})$ in three colors, say red, white, and blue, so that no line contains points of all three colors. To avoid trivialities we assume, of course, that each color is used at least once. One type of coloring can be obtained by coloring a point $p$ red, say, and coloring each line through $p$ (with $p$ deleted) either solid white or solid blue, randomly. Another type, essentially the dual of the above, is obtained by 
coloring the points on a line $l$ either white or blue, randomly, and coloring all points not on $l$ red. We shall call colorings of either of the above types trivial. Notice the following easily verified facts:

(a) the trivial colorings are precisely the ones in which some color is confined to a line.

(b) a coloring in which some line contains only one point of a given color must be trivial.

(c) a three-coloring of the points of $\boldsymbol{P}_{2}(F)$ leads to a dual threecoloring of the lines of $\boldsymbol{P}_{2}(F)$ by the pairs of colors $R W, R B$, or $W B$ of the points of the line. This coloring has the property that the lines of a pencil involve exactly two of those colors. Note that for the corresponding coloring of the affine plane not every line may be two-colored. Thus the dual coloring of the lines of the affine plane exists for the trivial coloring of the first kind in which no two colors are restricted to a single line, but not for the trivial coloring of the second kind.

(d) the two types of trivial colorings have in common colorings in which one point $p$ is red, say, all points on a line $l$ through $p$ are white (except $p$ ), and all points not on $l$ are blue. We call such a coloring a flag coloring.

We show that there is an intimate, and rather surprising, connection between colorings of $\boldsymbol{P}_{2}(F)$ and valuations on $F$ (see also [3, Theorem 3.7]).

THEOREM 1. The projective plane $\boldsymbol{P}_{2}(F)$ has a nontrivial 3coloring with no 3-colored lines if and only if the field $F$ has a nontrivial non-Archimedean valuation.

Before proceeding to the proof we mention several consequences.

CoRollary. $\boldsymbol{P}_{2}(F)$ has no nontrivial coloring if and only if $F$ is an algebraic extension of a finite field.

For a discussion of valuations see e.g., [5, XII, 4].

Hence $\boldsymbol{P}_{2}(\boldsymbol{R})$ has a nontrivial coloring whereas, for instance, finite projective planes do not. Our proof of Theorem 1 will in fact show that nontrivial colorings of $\boldsymbol{P}_{2}(\boldsymbol{F})$ are induced from trivial colorings of $\boldsymbol{P}_{2}(\bar{F})$ where $\bar{F}$ is the residue class field of $F$ with respect to some valuation. This, together with the nonconstructive nature of valuations on $\boldsymbol{R}$ yields the following.

CoRollary. All measurable colorings of $\boldsymbol{P}_{2}(\boldsymbol{R})$ are trivial, and 
the existence of nontrivial colorings cannot be proved without the Axiom of Choice.

No nontrivial valuation ring of $\boldsymbol{R}$ can be measurable, since $\boldsymbol{R}$ has no proper subgroup of positive measure. For the relation to the Axiom of Choice see e.g., [2].

We now proceed to the proof of Theorem 1 .

Proof. First, suppose that there is a nontrivial non-Archimedean valuation $x \rightarrow|x|$ on $F$. Let $\mathscr{O}=\{x|| x \mid \leqq 1\}$ be the associated valuation ring, $\mathscr{C}=\{x|| x \mid<1\}$ its maximal ideal, and $\bar{F}=\mathscr{O} / \mathscr{M}$ the residue class field. We color $\boldsymbol{P}_{2}(F)$ as follows:

Choose a homogeneous coordinate system $(x, y, z) \in F^{3}$ for $\boldsymbol{P}_{2}(F)$. Then color a point $p=(x, y, z)$ as follows:

(1) red if $|x|<|z|,|y|<|z|$

(2) white if $|x| \geqq|z|,|y|<|x|$

(3) blue if $|y| \geqq|z|,|y| \geqq|x|$.

It is easy to see that this gives a nontrivial 3-coloring of $\boldsymbol{P}_{2}(\boldsymbol{F})$. To see that no line is 3-colored suppose that $p_{i}=\left(x_{i}, y_{i}, z_{i}\right), i=1,2,3$, are points with $p_{1}$ red, $p_{2}$ white, and $p_{3}$ blue. Consider the determinant

$$
\left|\begin{array}{lll}
x_{1} & y_{1} & z_{1} \\
x_{2} & y_{2} & z_{2} \\
x_{3} & y_{3} & z_{3}
\end{array}\right|
$$

If $p_{1}, p_{2}, p_{3}$ were collinear then this determinant would be zero. However, from the colors of the $p_{i}$, it is clear that the term $z_{1} x_{2} y_{3}$ strictly dominates the other terms in the determinant expansion (with respect to the valuation), so the determinant cannot vanish ${ }^{1}$.

Notice that this coloring can be viewed as follows: choose a representative $(x, y, z)$ of $p$ with $x, y, z \in \mathcal{O}$ but not all of $x, y, z$ in $\mathscr{A}$. Then $(\bar{x}, \bar{y}, \bar{z}) \in \bar{F}^{3}$ corresponds to a point of $\boldsymbol{P}_{2}(\bar{F})$. The flag coloring of $\boldsymbol{P}_{2}(\bar{F})$ which colors the origin red, the rest of the $x$-axis white, and the rest of the plane blue, pulls back to the abovedescribed coloring of $\boldsymbol{P}_{2}(F)$. Similarly we can pull back any trivial coloring of $\boldsymbol{P}_{2}(\bar{F})$ to get a nontrivial coloring of $\boldsymbol{P}_{2}(\boldsymbol{F})$.

Now suppose that we are given a nontrivial coloring of $\boldsymbol{P}_{2}(F)$. We may choose coordinates so that the origin is red, the point at infinity on the $x$-axis is white, and the point at infinity on the $y$-axis is blue. Let $R_{x}=\{x \mid(x, 0,1)$ is colored red $\}$ and $R_{y}=\{y \mid(0, y, 1)$

${ }^{1}$ We recently became aware that this coloring was previously introduced in [6] for the affine plane. 
is colored red\}. Then if $(x, y, 1)$ is red the horizontal line through $(x, y, 1)$ is red-white so $(0, y, 1)$ is also red (since the $y$-axis is redblue). Hence $y \in R_{y}$. Similarly $x \in R_{x}$. Conversely, if $x \in R_{x}$ and $y \in R_{y}$ it is easy to see that $(x, y, 1)$ is red. Hence the red points are precisely $R_{x} \times R_{y}$.

Suppose now that the point $(b, 1,0)$ 'on the line at infinity is blue. If this point had originally been chosen to lie at infinity on the $y$-axis, the effect would have been that of a coordinate change $x \mapsto x-b y, y \mapsto y, z \mapsto z$. Hence, if $(x, y, 1)$ was originally red, $(x-b y, y, 1)$ is red in the new coordinates, so $(x-b y, 0,1)$ was originally red. Hence $x-b y \in R_{x}$. Conversely, if $x-b y \in R_{x}$ and $y \in R_{y}$ then $x \in R_{x}$. Hence $R_{x}$ is closed under translation by the subgroup $\left\langle b R_{y}\right\rangle$ generated by $b R_{y}$. Similarly, if $(1, w, 0)$ is white, $R_{y}$ is closed under translation by $\left\langle w R_{x}\right\rangle$. In particular, we have $\left\langle b R_{y}\right\rangle \subseteq R_{x}$ and $\left\langle w R_{x}\right\rangle \subseteq R_{y}$.

Now let $B_{z}=\{b \mid(b, 1,0)$ is blue $\}$ and $W_{z}=\{w \mid(1, w, 0)$ is white $\}$. Then from the above we have that the subring $\left\langle\left\langle B_{z} W_{z}\right\rangle\right.$ generated by $B_{z} W_{z}=\left\{b w \mid b \in B_{z}, w \in W_{z}\right\}$ maps $R_{x}$ into $R_{x}$ and $R_{y}$ into $R_{y}$ under multiplication. Let $\mathscr{O}_{z}=\left\{t \mid t\left\langle\left\langle B_{z} W_{z}\right\rangle \leqq\left\langle\left\langle B_{z} W_{z}\right\rangle\right\rangle\right\}\right.$. Then $\mathscr{O}_{z}$ is a subring of $F$. Furthermore $O_{z} \neq F$, since $\mathcal{O}_{z}=F$ would imply $\left\langle\left\langle B_{z} W_{z}\right\rangle=\right.$ $F$. (If $B_{z}$ or $W_{z}$ is $\{0\}$ then the coloring is trivial, by comment (b) early in this section.) But this, in conjunction with $\left.\left\langle B_{z} W_{z}\right\rangle\right\rangle R_{x} \leqq R_{x}$, implies that either $R_{x}=\{0\}$ or $R_{x}=F$, again forcing the coloring to be trivial. We now show that $O_{z}$ is a valuation ring, giving the desired valuation on $F$.

Suppose $t \in F$. If $t B_{z} \subseteq B_{z}$ then $t\left\langle\left\langle B_{z} W_{z}\right\rangle \leqq\left\langle B_{z} W_{z}\right\rangle\right\rangle$, so $t \in \mathcal{O}_{z}$. Otherwise, $t b \notin B_{z}$ for some $b \in B_{z}$, so $(t b, 1,0)$ is white, so $t^{-1} b^{-1} \in W_{z}$. Hence $t^{-1} \in B_{z} W_{z}$, so $t^{-1} \in \mathcal{O}_{z}$. This concludes the proof of Theorem 1 .

If we define $W_{x}=\{w \mid(1,0, w)$ is white $\}$ and $B_{y}=\{b \mid(0,1, b)$ is blue then in an exactly analogous way we get the nontrivial rings $\left\langle\left\langle R_{x} W_{x}\right\rangle\right\rangle$ and $\left\langle\left\langle R_{y} B_{y}\right\rangle\right\rangle$ and the corresponding valuation rings $\mathscr{O}_{x}$ and $O_{y}$ of $F$.

In order to prove that $\mathscr{O}_{x}=\mathcal{O}_{y}=\mathscr{O}_{z}$ we analyze the ring $\left\langle\left\langle B_{z} W_{z}\right\rangle\right.$ in more detail. We know that for each $b \in B_{z}, w \in W_{z}, b \neq 0$ we have $|w| \leqq\left|b^{-1}\right|$. If equality can occur then $\left\langle\left\langle B_{z} W_{z}\right\rangle\right\rangle$, being an $\mathcal{O}_{x}$ module and containing a unit of $\mathcal{O}_{z}$, must be $\mathcal{O}_{z}$. If equality does not occur then $\left\langle\left\langle B_{z} W_{z}\right\rangle\right\rangle=\mathfrak{M}_{z}$, the maximal ideal of $\mathcal{O}_{z}$. In either case $R_{x}$ and $R_{y}$ are closed under multiplication by $\mathfrak{M}_{z}$ and hence $\mathscr{O}_{x}$ and $\mathscr{O}_{y}$ are $\mathfrak{M}_{z}$-modules, but this implies that $\mathscr{O}_{x}=\mathscr{O}_{y}=\mathscr{O}_{z}=\mathscr{O}$.

Assume first that one of the rings $\left\langle\left\langle B_{z} W_{z}\right\rangle,\left\langle\left\langle R_{x} W_{x}\right\rangle\right\rangle,\left\langle\left\langle R_{y} W_{y}\right\rangle\right\rangle\right.$ is $\mathcal{O}$; say $\left\langle\left\langle B_{z} W_{z}\right\rangle=\mathcal{O}\right.$. Then there exist $b_{0} \in B_{z}, w_{0} \in W_{z}$ so that $\left|b_{0} w_{0}\right|=1$ so, by a change of scale $(x, y, 0)=\left(w_{0} x^{\prime}, w_{0} y^{\prime}, 0\right)$ we get $B_{z^{\prime}}, W_{z^{\prime}} \subseteq \mathcal{O}$.

Now $R_{x}, R_{y}$ are $\mathcal{O}$-modules and hence are given by a "Dedekind 
cut" in the valuation group $\Gamma$ corresponding to $\mathcal{O}$. That is, there exist $\alpha, \beta$ in the completion of $\Gamma$ so that

and

$$
R_{x}=\{x|x \in F,| x \mid<\alpha\} \text { or } R_{x}=\{x|x \in F,| x \mid \leqq \alpha\}
$$

$$
R_{y}=\{y|y \in F,| y \mid<\beta\} \text { or } R_{y}=\{y|y \in F,| y \mid \leqq \beta\} .
$$

If $\alpha, \beta \in \Gamma$ pick $u, v \in F$ so that $|u|=\alpha,|v|=\beta$ and make a change of scale $x=u x^{\prime}, y=v y^{\prime}$. Then $R_{x^{\prime}}=\mathfrak{M}$ or $\mathscr{O}$ and $R_{y^{\prime}}=\mathfrak{M}$ or $\mathcal{O}$. Correspondingly $W_{x^{\prime}}=\mathcal{O}$ or $\mathfrak{M}$ and $B_{y^{\prime}}=\mathcal{O}$ or $\mathfrak{M}$. Hence $\left\langle\left\langle R_{x^{\prime}}, W_{x^{\prime}}\right\rangle\right\rangle=\left\langle\left\langle R_{y^{\prime}} B_{y^{\prime}}\right\rangle\right\rangle=\mathfrak{M}$. Thus $R_{x^{\prime}}, R_{y^{\prime}}, W_{x^{\prime}}, B_{y^{\prime}}$ are unions of residue classes $(\bmod \mathfrak{M})$.

Now $W_{z^{\prime}}$ and $B_{z^{\prime}}$ are $(1+\mathfrak{M})$-closed. That is, $W_{z^{\prime}}+\mathfrak{M} W_{z^{\prime}}=W_{z^{\prime}}$, $B_{z^{\prime}}+\mathfrak{M} B_{z^{\prime}}=B_{z^{\prime}}$. Since, by our choice of scale we have $1 \in W_{z^{\prime}}$ and $b_{0} w_{0} \in B_{z^{\prime}}$ it follows that $B_{z^{\prime}}$ and $W_{z^{\prime}}$ are unions of residue classes $(\bmod \mathfrak{M})$.

Thus our coloring is a pull-back of a coloring of $\boldsymbol{P}_{2}(\bar{F})$. It is easy to see that the coloring of $\boldsymbol{P}_{2}(\bar{F})$ is trivial of the first type described when $R_{x^{\prime}}=R_{y^{\prime}}=\mathfrak{M}$ and of the second type when $R_{x^{\prime}}=$ $R_{y^{\prime}}=\mathscr{O}$.

In case $\left\langle\left\langle B_{z} W_{z}\right\rangle\right\rangle=\left\langle\left\langle R_{x} W_{x}\right\rangle\right\rangle=\left\langle\left\langle R_{y} B_{y}\right\rangle\right\rangle=\mathfrak{M}$ all the color classes are given by Dedekind cuts and in case these cuts are in $\Gamma$ we can change scales to make all the sets $R_{x}, R_{y}, W_{x}, W_{z}, B_{y}, B_{z}$ either $\mathfrak{M}$ or $O$ so that the coloring is again a pull-back of a trivial coloring of $\boldsymbol{P}_{2}(\bar{F})$.

We still need to consider the case in which the Dedekind cuts are not in $\Gamma$. In the first case we extend $F$ to a field $E$ by adjoining transcendentals $u$ or $v$ or both and extending the valuation by setting $|u|=\alpha,|v|=\beta$. The valuation ring $\mathcal{O}$ and its maximal ideal $\mathfrak{M}$ are then extended to $\mathcal{O}_{E}$ and $\mathfrak{M}_{E}$ and the coloring of $\boldsymbol{P}_{2}(F)$ is extended to that of $\boldsymbol{P}_{2}(E)$ by making the sets $R_{x}, R_{y}, W_{x}, B_{y}$ equal to $\mathfrak{M}_{E}$ or $\mathscr{O}_{E}$. The extension of $B_{z}$ and $W_{z}$ may not be unique, but we can set

$$
\begin{aligned}
& W_{z, E}=\left(W_{z} \cup\{w|| w \mid=1, w \notin F\}\right)\left(1+\mathfrak{M}_{E}\right) \\
& B_{z, E}=B_{z}+B_{z} \mathfrak{M}_{E} .
\end{aligned}
$$

This coloring is a pull-back of a trivial coloring of $\boldsymbol{P}_{2}(\bar{E})=\boldsymbol{P}_{2}(\bar{F})$ and it induces a coloring on the subspace isomorphic to $\boldsymbol{P}_{2}(F)$.

The extension, if necessary, in case $\left\langle\left\langle B_{z} W_{z}\right\rangle\right\rangle=\left\langle\left\langle R_{x} W_{x}\right\rangle\right\rangle=\left\langle\left\langle R_{y} B_{y}\right\rangle\right\rangle=$ $\mathfrak{M}$ is entirely analogous.

To sum up:

THEOREM 2. Every 3-coloring of $\boldsymbol{P}_{2}(F)$ defines a unique non- 
Archimedean valuation $v$ on $F$ and the coloring of $\boldsymbol{P}_{2}(F)$ is a pull-back of a trivial coloring (corresponding to the trivial valuation) on $\boldsymbol{P}_{2}(\bar{F})$ where $\bar{F}$ is the residue class field determined by $v$. This pull-back may be obtained via an embedding of $\boldsymbol{P}_{2}(F)$ in $\boldsymbol{P}_{2}(E)$ where $E$ is a (finitely generated) extension field of $F$.

REMARK. In every 4-coloring of $\boldsymbol{P}_{2}(F)$ there must occur 3-colored lines, regardless of the nature of $F$. To see this, let $p, q, r, s$ be red, white, blue and yellow respectively. Since the lines $p q$ and $r s$ intersect at least one of them is three-colored.

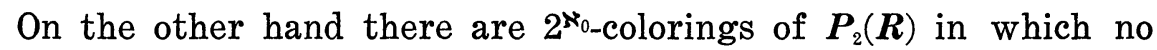
line has more than three colors. For example color the points of a strictly convex curve with distinct colors other than red and all other points red.

3. Higher dimensions. We now consider colorings of projective $n$-space $\boldsymbol{P}_{n}(\boldsymbol{F})$ in $n+1$ colors so that no hyperplane contains all $n+1$ colors. As before we consider colorings to be trivial if any color is confined to a hyperplane. One such trivial coloring can be obtained by generalizing our previous flag colorings in an obvious way: color a point red, the rest of a line through that point white, the rest of a plane through that line blue, etc. Then we have the following extension of our previous result.

TheOREM 3. The projective space $\boldsymbol{P}_{n}(F)$ has a nontrivial $n+1-$ coloring with no $n+1$-colored hyperplane if and only if the field $F$ has a nontrivial valuation.

Proof. Suppose $x \rightarrow|x|$ is a valuation with associated $\mathcal{O}, \mathscr{C}$, and $\bar{F}$ as before. We obtain a nontrivial coloring of $\boldsymbol{P}_{n}(F)$ by pulling back a generalized flag coloring of $\boldsymbol{P}_{n}(\bar{F})$. More specifically, we color $\left(x_{1}, x_{2}, \cdots, x_{n+1}\right)$ red if $\left|x_{i}\right|<\left|x_{n+1}\right|$ for $i<n+1$, white if $\left|x_{1}\right| \geqq\left|x_{n+1}\right|$ and $\left|x_{i}\right|<\left|x_{1}\right|$ for $2 \leqq i \leqq n$, blue if $\left|x_{2}\right| \geqq\left|x_{n+1}\right|,\left|x_{2}\right| \geqq$ $\left|x_{1}\right|$, and $\left|x_{i}\right|<\left|x_{2}\right|$ for $3 \leqq i \leqq n$, etc. Then a determinant argument as in $\S 2$ shows that no hyperplane is $n+1$-colored.

Now suppose that a nontrivial coloring exists. Choose $n$ points involving $n$ colors and pass a hyperplane through them. This gives an $n$-coloring of $\boldsymbol{P}_{n-1}(F)$. Furthermore no hyperplane of this $\boldsymbol{P}_{n-1}(F)$ contains all $n$ colors, indeed, no $k$-plane can be $(k+2)$-colored $(k=1, \cdots, n-1)$, since then it could be enlarged to a hyperplane of $\boldsymbol{P}_{n}(\boldsymbol{F})$ containing $n+1$ colors. Hence, by induction on $n$, either this coloring of $\boldsymbol{P}_{n-1}(F)$ is trivial or $F$ has a nontrivial valuation. It only remains to show that, if the $n$-coloring of $\boldsymbol{P}_{n-1}(F)$ is trivial, then the $n+1$-coloring of $\boldsymbol{P}_{n}(\boldsymbol{F})$ can't be nontrivial. (This is 
really an extension of comment (b) of $\S 2$.) Suppose, for instance, that the color red is confined to an $n-2$ dimensional subspace of $\boldsymbol{P}_{n-1}(F)$. Pick a red point $p$ on this $n-2$ dimensional subspace and a line $l$ through $p$ in $\boldsymbol{P}_{n-1}(F)$ but not in the $n-2$ dimensional subspace. Then this line $l$ is solid white, say, except for $p$. Choose now $n-1$ points involving all colors except red and white. Then any hyperplane through these points and a red point must cut $l$ in $p$ but not contain $l$ (or it would involve all $n+1$ colors), so these $n-1$ points and $p$ determine a hyperplane containing all red points. This contradicts the nontriviality of the original coloring.

This completes the proof of Theorem 3. A more detailed analysis, which we omit, shows that any nontrivial coloring is "essentially" a pull back of a trivial coloring of $\boldsymbol{P}_{n}(\bar{F})$, as in the planar case.

4. Linear systems of curves. Suppose one wishes to color the projective plane $\boldsymbol{P}_{2}(F)$ in six colors so that no conic contains more than five colors. As before we insist that no color is confined to a conic to avoid trivialities. Our previous results enable us to do this, and in fact to do something much more general.

Consider $n+1$ algebraic curves in $\boldsymbol{P}_{2}(F)$ which are the loci of $n+1$ homogeneous algebraic equations

$$
f_{i}(x, y, z)=0 ; i=0,1,2, \cdots, n
$$

where $f_{i} \in F[x, y, z]$ are polynomials of the same degree and linearly independent over $F$. These curves define an $n$-dimensional linear system of curves, the loci of

$$
c_{0} f_{0}+c_{1} f_{1}+\cdots+c_{n} f_{n}=0,
$$

where $\left(c_{0}, c_{1}, \cdots, c_{n}\right) \in \boldsymbol{P}_{n}(F)$.

THEOREM 4. Let $C$ be an n-dimensional linear system of algebraic curves in $\boldsymbol{P}_{2}(\boldsymbol{F})$, such that no point in $\boldsymbol{P}_{2}(\boldsymbol{F})$ lies on all curves in $C$. If $F$ admits a nontrivial valuation then there is a coloring of $\boldsymbol{P}_{2}(F)$ in $n+1$ colors so that no curve in $C$ contains all $n+1$ colors and no color is confined to a curve in $C$.

Proof. By duality points of $\boldsymbol{P}_{2}(F)$ can be regarded as (one dimensional subspaces of) linear functions on $C$. In other words there is a map $\phi$ of $\boldsymbol{P}_{2}(F)$ into the $n$-dimensional projective space $\boldsymbol{P}\left(C^{*}\right)$ determined by the dual space $C^{*}=\operatorname{Hom}_{F}(C, F)$ of $C$. But we can color $\boldsymbol{P}\left(C^{*}\right)$ in $n+1$ colors using a valuation as in Theorem 3. This will give an $(n+1)$-coloring of $\boldsymbol{P}\left(C^{*}\right)$ which we restrict to $\phi\left(\boldsymbol{P}_{2}(F)\right.$ ), giving a coloring of $\boldsymbol{P}_{2}(F)$. Since curves in $C$ correspond to hyperplanes in $\boldsymbol{P}\left(C^{*}\right)$, no curve in $C$ will contain all $n+1$ colors. 
All that remains is to show that the coloring can be chosen nontrivial on $\phi\left(\boldsymbol{P}_{2}(\boldsymbol{F})\right)$. In fact, we must first arrange that all $n+1$ colors appear on $\phi\left(\boldsymbol{P}_{2}(F)\right)$, and then nontriviality will follow. To do this choose points $p_{0}, p_{1}, p_{2}, \cdots, p_{n}$ in $\boldsymbol{P}_{2}(\boldsymbol{F})$ whose images under $\phi$ do not lie in a hyperplane of $\boldsymbol{P}\left(C^{*}\right)$; this can be done since no curve in $C$ contains all of $\boldsymbol{P}_{2}(F)$-remember $F$ cannot be finite! Then coordinatize $\boldsymbol{P}\left(C^{*}\right)$ with respect to the $n+1$ points $\phi\left(p_{i}\right)$ and use as the coloring of $\boldsymbol{P}\left(C^{*}\right)$ a pull back of a generalized flag coloring with respect to these coordinates. This guarantees that $p_{0}, p_{1}, \cdots, p_{n}$ are all colored differently, so the proof is complete.

Theorem 4 can clearly be generalized to linear systems of hypersurfaces in higher dimensions. In view of Theorems 1 and 3, it is also tempting to conjecture that the converse of Theorem 4 holds, i.e., that the existence of a nontrivial coloring with respect to a linear system implies the existence of a nontrivial valuation on $F$. If a coloring of $\boldsymbol{P}_{2}(F)$ is given we can assign to each curve in $C$ one of the colors not on it, obtaining a "dual" coloring of $\boldsymbol{P}(C)$. We cannot apply Theorem 3 to this coloring of $\boldsymbol{P}(C)$ to get a valuation unless we know that all hyperplanes of $\boldsymbol{P}(C)$ omit at least one color, and our hypotheses only guarantee this for hyperplanes of $\boldsymbol{P}(C)$ corresponding to points of $\boldsymbol{P}\left(C^{*}\right)$ that lie in $\phi\left(\boldsymbol{P}_{2}(F)\right)$. Without an 'ad hoc' hypothesis to cover the other points we do not see how to proceed.

5. The fundamental theorem of projective geometry. Colorings of $\boldsymbol{P}_{2}(\boldsymbol{F})$ in three colors can be considered as mappings from $\boldsymbol{P}_{2}(F)$ to the 3-point projective plane

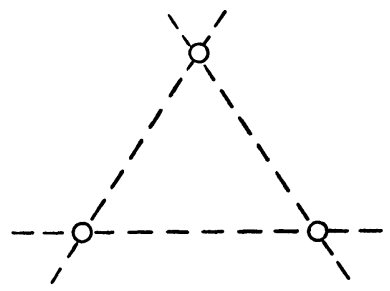

which preserve collinearity. (One can consider this as the projective plane over the "one-element field", since $n^{2}+n+1=3$ when $n=1$ ). Furthermore the colorings we use in Theorem 1 are obtained via mappings from $\boldsymbol{P}_{2}(F)$ to $\boldsymbol{P}_{2}(\bar{F})$ which preserve collinearity. These observations suggest the validity of an extension of the Fundamental Theorem of Projective Geometry (FTPG) ${ }^{2}$. There are many versions

${ }^{2}$ In fact it is a question of this kind which appears to have motivated Bognár's original question (private written communication). 
of the FTPG in the literature. The one we refer to is essentially that of Artin's [1]. We state it here for projective planes over fields only, and in non-coordinate-free notation:

(FTPG): Let $\phi$ be a $1-1$ mapping from $\boldsymbol{P}_{2}\left(\boldsymbol{F}_{1}\right)$ onto $\boldsymbol{P}_{2}\left(\boldsymbol{F}_{2}\right)$ which preserves collinearity. Then there is an isomorphism $\mu$ from $F_{1}$ onto $F_{2}$ and $a 3 \times 3$ invertible matrix $M$ over $F_{2}$ so that $\phi$ is induced by the mapping from $F_{1}^{3}$ to $F_{2}^{3}$ given by $(x, y, z) \mapsto\left(x^{\mu}, y^{\mu}, z^{\mu}\right) M$. Furthermore $\phi$ determines $\mu$ uniquely, and determines $M$ up to multiplication by a scalar matrix.

The extension of this theorem we give allows $\phi$ to be neither $1-1$ nor onto. In a sense we classify all morphisms in the appropriate category whereas the FTPG classifies only isomorphisms.

THEOREM 5. Let $\phi$ be a mapping from $\boldsymbol{P}_{2}\left(\boldsymbol{F}_{1}\right)$ to $\boldsymbol{P}_{2}\left(\boldsymbol{F}_{2}\right)$ which preserves collinearity, and whose image contains four points, no three collinear. Then there is a place $\mu$ from $F_{1}$ into $F_{2}$ and invertible $3 \times 3$ matrices $M_{1}, M_{2}$ over $F_{1}, F_{2}$ respectively, so that $\phi$ is induced by the map from $F_{1}^{3}$ to $F_{2}^{3}$ given by $\vec{v}=(x, y, z) \mapsto\left(\vec{v} M_{1}\right)^{\mu} M_{2}$.

(Here $(x, y, z)^{\mu}$ means the vector obtained by scaling $(x, y, z)$ to have integral coordinates, not all in the maximal ideal, and then applying $\mu$ to each coordinate.)

Proof. By a change of coordinates in $\boldsymbol{P}_{2}\left(\boldsymbol{F}_{2}\right)$ we may assume that the four points, no three collinear, in the image of $\phi$ are $(0,0,1),(1,0,0),(0,1,0)$, and $(1,1,1)$. (In affine coordinates the origin $(0,0)$, the points at $\infty$ on the $x$ and $y$ axes, and $(1,1))$. This change of coordinates gives the matrix $M_{2}$. Notice that one of the standard methods for introducing coordinates in a projective plane [4] applied to $\phi\left(\boldsymbol{P}_{2}\left(F_{1}\right)\right)$ now shows that $\left\{t \mid(t, t, 1) \in \phi\left(\boldsymbol{P}_{2}\left(\boldsymbol{F}_{1}\right)\right)\right\}$ is a subfield $F_{2}^{\prime}$ of $F_{2}$ and $\phi\left(\boldsymbol{P}_{2}\left(F_{1}\right)\right)$ consists precisely of points whose coordinates lie in $F_{2}^{\prime}$, i.e., a copy of $\boldsymbol{P}_{2}\left(F_{2}^{\prime}\right)$.

Now, by a change of coordinates in $P_{2}\left(F_{1}\right)$, we may assume that $\phi$ maps $(0,0,1)$ to $(0,0,1),(1,0,0)$ to $(1,0,0),(0,1,0)$ to $(0,1,0)$, and $(1,1,1)$ to $(1,1,1)$. This change of coordinates gives the matrix $M_{1}$. It only remains to be shown that, with respect to the new coordinates, $\phi$ is induced by $(x, y, z) \mapsto(x, y, z)^{\mu}$ for some place $\mu$.

Let $\mathscr{O}=\{t \mid \phi(t, t, 1) \neq(1,1,0)\}$, i.e., the coordinates of points on the line $y=x$ in $\boldsymbol{P}_{2}\left(F_{1}\right)$ that are not mapped by $\phi$ to a point at infinity. Define $\mu: F_{1} \rightarrow F_{2}$ by $\mu(t)=\infty$ if $t \notin \mathcal{O}$, and $\mu(t)=t^{\prime}$ if $t \in \mathcal{O}$ and $\phi(t, t, 1)=\left(t^{\prime}, t^{\prime}, 1\right)$. We claim that $\mu$ is a place and that $\mu$ induces $\phi$. 
The argument here is essentially the one used in introducing coordinates in a plane, namely that addition and multiplication are determined by geometric configurations. First, observe that by considering horizontal and vertical lines and their intersections we obtain that the image of a "finite" point $\left(x_{0}, y_{0}\right)$ under $\phi$ is finite if and only if $x_{0}$ and $y_{0}$ lie in $\mathcal{O}$. Furthermore the point $(1,1,0)$ is mapped to $(1,1,0)$ by $\phi$. If $t_{1}, t_{2}$ lie in $\mathcal{O}$ then the line $y=x+t_{1}$ determined by $\left(0, t_{1}, 1\right)$ and $(1,1,0)$ is mapped into a line not at $\infty$ by $\phi$, so the intersection of this line with $y=t_{2}$ is mapped to a finite point. In other mords $\left(t_{2}-t_{1}, t_{2}, 1\right)$ is mapped to a finite point, so $\mathcal{O}$ is closed under subtraction. Furthermore the configuration used in this argument is preserved under $\phi$, so we must have $\left(t_{2}-t_{1}\right)^{\mu}=t_{2}^{\mu}-t_{1}^{\mu}$ also.

The line $y=t_{1} x$ determined by $(0,0,1)$ and $\left(1, t_{1}, 1\right)$ is mapped under $\phi$ into the line determined by $(0,0,1)$ and $\left(1, t_{1}^{\mu}, 1\right)$. Hence, the intersection of this line with $x=t_{2}$ is mapped to a finite point, i.e., $\left(t_{2}, t_{1} t_{2}, 1\right)$ is mapped to a finite point. Hence $\mathcal{O}$ is closed under multiplication, and as before we also obtain $\left(t_{1} t_{2}\right)^{\mu}=t_{1}^{\mu} t_{2}^{\mu}$.

We now have $\mu: \mathcal{O} \rightarrow F_{2}$ being a ring homomorphism with kernel $\mathscr{C}=\{t \mid \phi(t, t, 1)=(0,0,1)\}$ and image the subfield $F_{2}^{\prime}$ of $F_{2}$ alluded to earlier. Suppose that $t \in F_{1}, t \neq 0$. Then the points $(0,0,1),(1, t, 1)$, and $\left(t^{-1}, 1,1\right)$ are collinear, lying on $y=t x$. Then their images must be collinear, so $t$ and $t^{-1}$ cannot both lie outside $\mathcal{O}$ (or their images would be $(0,0,1),(0,1,0),(1,0,0)$ ). Hence $\mathcal{O}$ is a valuation ring, and $\mu$ is the associated place. Furthermore it is now clear that $\mu$ determines the mapping $\phi$, so the proof is complete.

The extension of Theorem 5 to higher dimensions is completely straightforward. We omit the details.

A coordinate-free restatement of Theorem 5 would look something like this: Let $V_{1}$ and $V_{2}$ be 3-dimensional vector spaces over $F_{1}$ and $F_{2}$, and let $\phi: P\left(V_{1}\right) \rightarrow \boldsymbol{P}\left(V_{2}\right)$ preserve collinearity and contain 4 points, no 3 collinear, in its image. Then there is a valuation of $F_{1}$, a free rank $3 \mathcal{O}$-submodule $W$ of $V_{1}$, an embedding $\mu: \mathcal{O} / \mathscr{M} \rightarrow$ $F_{2}$, and an $F_{2}$-isomorphism from $(W / \mathscr{C} W) \otimes_{O / \mathscr{M}} F_{2}$ onto $V_{2}$ which induces $\phi$. It is unfortunate that $W$ need be specified, but this appears unavoidable.

Finally, a word about the uniqueness of the ingredients in Theorem 5. It is not difficult to show that $\phi$ uniquely determines the place $\mu$. However, $M_{1}$ and $M_{2}$ are not uniquely determined up to multiplication by scalar matrices. We must also allow for replacing $M_{1}$ and $M_{2}$ by $\left(M_{1} M^{-1}\right)$ and $\left(M^{\mu} M_{2}\right)$ where $M$ is an invertible (with respect to $\mathcal{O}$ ) $3 \times 3$ matrix with entries in $\mathcal{O}$. 
6. Divisibility of quadrilaterals into disjoint triangles of equal areas. Monsky [6] applied the coloring described in $\S 2$ to the affine plane $A_{2}(\boldsymbol{R})$. That is, the point $(x, y)$ is

(1) red if $|x|<1,|y|<1$

(2) white if $|x| \geqq 1,|y|<|x|$

(3) blue if $|y| \geqq 1,|y| \geqq|x|$.

The argument for the noncollinearity of three points of different color in the proof of Theorem 1 then yields that the area, $A$, of a triangle with vertices of different colors (3-colored triangle for short) satisfies

$$
|A|=\left|\frac{1}{2}\right| \begin{array}{lll}
x_{1} & y_{1} & 1 \\
x_{2} & y_{2} & 1 \\
x_{3} & y_{3} & 1
\end{array}|| \geqq\left|\frac{1}{2}\right| .
$$

Using the fact that the vertices $(0,0),(1,0),(1,1),(0,1)$ of the unit square are colored $R W B B$ so that the square has, say, a single $R W$ edge, a simple combinatorial argument shows that every triangulation of the unit square must contain a 3-colored triangle. A 2-adic valuation of $\boldsymbol{R}$ shows that if the area of such a triangle is the reciprocal of an integer, that integer must be even. In other words the unit square cannot be divided into an odd number of disjoint triangles (that is, triangles with disjoint interiors) of equal areas.

We now can ask the question: which other quadrilaterals have the property that they cannot be divided into an odd number of disjoint triangles of equal areas? Since this property is an affine invariant we may assume that three of the vertices are $(0,0),(1,0)$, $(0,1)$ and let the fourth vertex be $(x, y), x \geqq y$.

If there exists a 2-adic valuation of $R$ for which $\max \{|x|,|y|\} \geqq$ 1 then the vertex $(x, y)$ is not colored red. Thus any triangulation must involve a 3-colored triangle and if the area of the quadrilateral satisfies

$$
|(x+y) / 2|<2 \leqq \mid \text { area of 3-colored triangle } \mid
$$

then the number of triangles must be even. All of these conditions are satisfied if $x, y$ are odd rational numbers. We have thus proved the following:

THEOREM 6. The set of quadrilaterals which cannot be divided into an odd number of disjoint triangles of equal areas is everywhere dense.

Using any $p$-adic valuation, $p>2$, and $(x, y)$ with $\max \{|x|,|y|\} \geqq$ 
$1,|(x+y) / 2|<1 \leqq \mid$ area of 3 -colored triangle $\mid$, which is satisfied by $p$-adically integral rationals $x, y$ with $x+y \equiv 0(\bmod p)$, we get a more general result.

THEOREM 7. For any prime $p$ there exists an everywhere dense set of quadrilaterals which cannot be divided into $k$ disjoint triangles of equal areas unless $k$ is divisible by $p$.

We now observe that the set of quadrilaterals which can be divided into $k$ disjoint triangles of equal areas is clearly a closed set. To see this let the fourth vertex $\left(x_{n}, y_{n}\right)$ approach $\left(x_{0}, y_{0}\right)$. Then for a subsequence the vertices of the dividing triangles $\left(x_{n, i}, y_{n, i}\right)$ converge to vertices $\left(x_{0, i}, y_{0, i}\right)$ and for a subsequence of these the corresponding triangulations converge. Since Theorem 7 shows that this closed set is nowhere dense, we get a surprising corollary.

THEOREM 8. The set of quadrilaterals which can be divided into a number of disjoint triangles of equal areas is of the first category.

The restriction to quadrilaterals was only for the convenience of exposition and Theorem 8 holds for $n$-gons with $n \geqq 4$. It seems that the vertices $(x, y)$ for which the quadrilateral can be divided into $k$ triangles of equal areas lie on the union of a finite number of algebraic curves and thus form a set of measure 0 . Thus the set in Theorem 8 would also have measure 0 .

There are no analogous results for the division of polygons into disjoint quadrilaterals of equal areas. In fact it is quite easy to see that any polygon can be divided into a finite number of quadrilaterals of equal areas.

Generalizations to the division of polyhedra into disjoint simplices of equal volumes are possible, but in addition to the colorings discussed in $\S 3$ we need generalizations of Monsky's combinatorial lemma on triangulations. We therefore do not go into the details.

\section{REFERENCES}

1. E. Artin, Geometric Algebra, Interscience Publishers, New York, 1957.

2. J. Briggs and T. Schaffter, Measure and cardinality, Amer. Math. Monthly, 86 (1979), 852-855.

3. D. S. Carter and A. Vogt, Collinearity-Preserving Functions Between Desarguesian Planes, AMS Memoir No. 235.

4. M. Hall, Projective planes and related topics, California Institute of Technology, 1954.

5. S. Lang, Algebra, Addison-Wesley, 1965. 
6. P. Monsky, On dividing a square into triangles, Amer. Math. Monthly, (1970), 161-164.

Received September 2, 1980 and in revised form May 15, 1981. Work of the second author has been supported in part by NSF Grant MCS 79-03162.

UNIVERSITY OF CALIFORNIA

LOS ANGELES, CA 90024 



\section{PACIFIC JOURNAL OF MATHEMATICS}

\section{EDITORS}

DONALD BABBITT (Managing Editor)

University of California

Los Angeles, California 90024

\section{Hugo Rossi}

University of Utah

Salt Lake City, UT 84112

C. C. MOore and Arthur AGuS

University of California

Berkeley, CA 94720
J. DugundJI

Department of Mathematics University of Southern California Los Angeles, California 90007

R. FinN and J. Milgram Stanford University Stanford, California 94305

ASSOCIATE EDITORS
R. ARENS
E. F. BECKENBACH
B. H. NeumanN
F. WOLF
K. YOSHIDA

\section{SUPPORTING INSTITUTIONS}

UNIVERSITY OF ARIZONA

UNIVERSITY OF BRITISH COLUMBIA

CALIFORNIA INSTITUTE OF TECHNOLOGY

UNIVERSITY OF CALIFORNIA

MONTANA STATE UNIVERSITY

UNIVERSITY OF NEVADA, RENO

NEW MEXICO STATE UNIVERSITY

OREGON STATE UNIVERSITY
UNIVERSITY OF OREGON

UNIVERSITY OF SOUTHERN CALIFORNIA

STANFORD UNIVERSITY

UNIVERSITY OF HAWAII

UNIVERSITY OF TOKYO

UNIVERSITY OF UTAH

WASHINGTON STATE UNIVERSITY

UNIVERSITY OF WASHINGTON 


\section{Pacific Journal of Mathematics}

Vol. 99, No. $1 \quad$ May, 1982

Mariano Giaquinta, Jindrich Necas, O. John and J. Stará, On the

regularity up to the boundary for second order nonlinear elliptic systems . . 1

Siegfried Graf, Realizing automorphisms of quotients of product $\sigma$-fields . . 19

Alfred Washington Hales and Ernst Gabor Straus, Projective colorings . . . 31

Sandra Hayes, The weak Nullstellensatz for finite-dimensional complex

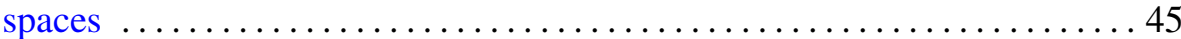

Gerald Norman Hile and Murray Harold Protter, The Cauchy problem

and asymptotic decay for solutions of differential inequalities in Hilbert

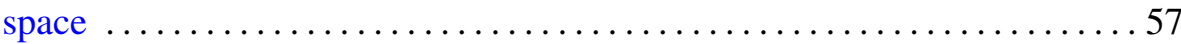

Robert D. Little, Projective space as a branched covering with orientable

branch set ......................................... 89

Jaroslav Mach, On the proximinality of Stone-Weierstrass subspaces . . . . . 997

John C. Morgan, II, On product bases ...................... 105

K. Balakrishna Reddy and P. V. Subrahmanyam, Altman's contractors

and fixed points of multivalued mappings . .................. 127

James Ted Rogers Jr., Decompositions of homogeneous continua . . . . . . . 137

Ahmed Ramzy Sourour, Characterization and order properties of

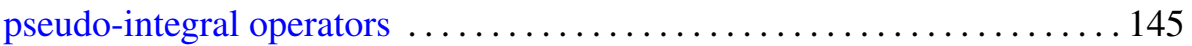

Robert Moffatt Stephenson Jr., Pseudocompact and Stone-Weierstrass

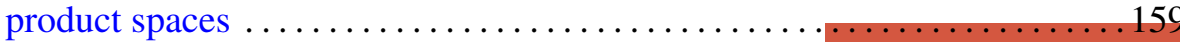

Bruce Stewart Trace, On attaching 3-handles to a 1-connected

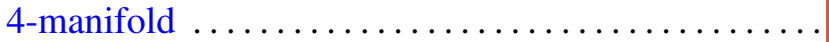

Akihito Uchiyama, The construction of certain BMO functions and the corona problem

Thomas Alva Whitehurst, An application of orthogonal polynomials to random walks ..............................

David J. Winter, Root locologies and idempotents of Lie and nonassociative algebras

William Robin Zame, The classification of uniform algebras on plane domains 